\title{
Recent Advances in Experimental Studies of Steady-State Dilution and Reactive Mixing in Saturated Porous Media
}

\author{
Tiantian Xu, Yu Ye *, Yu Zhang and Yifan Xie \\ State Key Laboratory of Hydrology-Water Resources and Hydraulic Engineering, Hohai University, \\ Nanjing 210098, China; xtt@hhu.edu.cn (T.X.); yuzhang@hhu.edu.cn (Y.Z.); yfxie@hhu.edu.cn (Y.X.) \\ * Correspondence: yeyu@hhu.edu.cn; Tel.: +86-137-7077-4020
}

Received: 12 November 2018; Accepted: 19 December 2018; Published: 20 December 2018

\begin{abstract}
Transverse dispersive mixing plays an important role in controlling natural attenuation of contaminant plumes and the performance of engineered remediation strategies. The extent of transverse mixing can be significantly affected by porous media heterogeneity and anisotropy. For instance, flow focusing in the high-permeability inclusions leads to an enhancement of dilution and reactive mixing in steady-state solute transport. Numerous modeling studies have been performed to understand the mechanism of conservative and reactive transport in homogeneous and complex heterogeneous porous media. However, experimental investigations are necessary to show an intuitive phenomenon and to validate the modeling results. This paper briefly reviews recent laboratory experimental studies on dilution and reactive mixing of steady-state transport in saturated homogeneous and heterogeneous porous media. In this context, setups and measuring techniques are described in pore-scale and Darcy-scale experiments. Parameters quantifying dilution and reactive mixing in the experiments are also introduced. Finally, we discuss the further experimental works necessary to deepen our understanding of dilution and reactive mixing in natural aquifers.
\end{abstract}

Keywords: dilution; reactive mixing; experiments; heterogeneity; porous media

\section{Introduction}

The study of dilution and reactive mixing in porous media is of pivotal importance in a variety of scientific fields (e.g., contaminant hydrology, reservoir engineering) and technical applications (e.g., groundwater treatment, nuclear waste storage). In groundwater systems, the degradation of contaminant plumes is determined by the interplay between physical and biogeochemical processes. In particular, insufficient mixing of electron donors (e.g., chemicals of the contaminant plumes) and electron acceptors (e.g., dissolved oxygen of the ambient groundwater) often limits (bio)chemical reactions, thus controlling natural attenuation of the contaminant plumes and the performance of engineered remediation strategies.

In general, mixing is a term used in the reactive transport, while dilution is a term used in the conservative transport. Dilution is also a physical process leading to the reduction of the peak concentration and the increase of the plume entropy. In fact, both dilution and mixing of the plumes are caused by dispersion, and the extents of both processes are affected by the heterogeneity and anisotropy of the porous media [1-3].

The quasi steady-state contaminant transport has been found in field investigations. In these circumstances, the contaminants are released continuously from the source such as NAPL (Non-aqueous Phase Liquid) spills or leaking landfills [4,5]. The steady-state conditions are approached when the equilibrium between the release and the degradation of the contaminant mass is reached. 
At steady-state conditions, transverse dispersive mixing is the key process determining the length of the plume and the fate of the contaminant transport [6-11]. Even though the transverse dispersion coefficient is smaller than the longitudinal dispersion coefficient, contact areas between the mixing solutions are much larger in the transverse direction compared to the longitudinal direction. Only at the front of the plume does longitudinal dispersion play an important role in steady-state plume transport. This can be reflected by the steep concentration gradients found at the fringes of groundwater plumes in the field investigations of contaminant aquifers [12-15].

Observation and measuring points in the field investigation of contaminant transport are generally coarse. Furthermore, the heterogeneity and anisotropy of porous media and the biogeochemical complexity of natural aquifer systems influence dilution and mixing. The interpretation separating different physical and chemical processes and the influencing factors is difficult in natural groundwater systems. A detailed analysis of the pure transverse dispersive mixing process and its influencing factors under well-defined and controlled conditions is only possible by performing laboratory experiments and numerical simulations. Numerous modeling studies have been conducted to investigate transverse dispersion of conservative and reactive solutes in homogeneous and heterogeneous porous media [16-19]. However, a major challenge of various numerical methods is their numerical dispersion and the resulting uncertainties. Therefore, laboratory experiments are always necessary to give an intuitive view of the phenomenon and to test and verify the numerical models.

The objectives of this paper are to give a brief review of two-dimensional and three-dimensional experiments studying steady-state conservative and reactive transport in saturated porous media, discuss their effectiveness and limitations, and review the influence of the heterogeneity and anisotropy of porous media arrangements on solute transport. This paper also tries to synthesize the approaches used to quantify dilution and reactive mixing of steady-state plumes.

This brief review does not intend to exhaustively review all the experimental works in the field of steady-state dilution and reactive mixing in saturated porous media. In contrast, we focus on the recent advances in the experimental works studying dilution and reactive mixing.

\section{Theory}

The description of steady-state flow depends on the scale of considered experiments. At the pore scale, flow can be calculated by the combination of the continuity equation and the Navier-Stokes equation. The latter can be written as:

$$
-\rho g \nabla \phi+\mu \nabla^{2} \mathbf{u}=0
$$

where $\rho$ is the fluid density, $g$ is the constant of acceleration due to gravity, $\phi$ is the hydraulic head, $\mu$ is the dynamic viscosity, and $\mathbf{u}$ is the velocity vector. The first term at the left part of Equation (1) turns out to be the pressure gradient. At the Darcy scale, the combination of the continuity equation and the Darcy's law leads to the governing steady-state flow equation in saturated porous media:

$$
\nabla \cdot(\mathbf{q}(\mathbf{x}))=\nabla \cdot(-\mathbf{K}(\mathbf{x}) \nabla \phi(\mathbf{x}))=0
$$

where $\mathbf{q}$ is the specific discharge vector, $\mathbf{x}$ is the vector of spatial coordinates, and $\mathbf{K}$ is the hydraulic conductivity tensor with the expression depending on the porous media (i.e., isotropic or anisotropic) and the orientation of the Cartesian coordinate system.

At the pore scale, steady-state solute transport can be described by the advectiondiffusion-reaction equation:

$$
\mathbf{u} \cdot \nabla c-\nabla \cdot\left(D_{a q} \nabla c\right)=r
$$


where $c$ is the concentration, $D_{a q}$ is the aqueous diffusion coefficient, and $r$ is the reaction rate which equals zero for conservative solute transport. At the Darcy scale, advection-dispersion-reaction equation describes solute transport. For steady-state reactive transport, such an equation reads as:

$$
\mathbf{v} \cdot \nabla c-\nabla \cdot(\mathbf{D} \nabla c)=r
$$

where $\mathbf{v}$ is the seepage velocity vector (i.e., $\mathbf{v}=\mathbf{q} / \theta), \theta$ is the porosity, and $\mathbf{D}=\frac{\mathbf{v} \otimes \mathbf{v}}{\mathbf{v} \cdot \mathbf{v}}\left(D_{l}-D_{t}\right)+\mathbf{I} D_{t}$ is the dispersion tensor, $v \otimes v$ and $\mathbf{v} \cdot \mathbf{v}$ are the tensor and scalar product of $\mathbf{v}$ itself, $D_{l}$ and $D_{t}$ are the longitudinal and transverse dispersion coefficient, and $\mathbf{I}$ is the identity matrix. For steady-state transport of continuously emitted plumes, transverse dispersion is of key importance [18]. Therefore, the transverse dispersion coefficient (i.e., $D_{t}$ ) is highly relevant to the resulted concentration distributions in the experiments [9].

The transverse dispersion coefficient is defined as the additive contribution of a pore diffusion coefficient (i.e., $D_{p}$ ) and a mechanical dispersion coefficient (i.e., $D_{\text {mech }}$ ). The pore diffusion coefficient is often derived from empirical correlations $[20,21]$ since molecular diffusion can only occur in pore spaces and the size of the pores as well as the tortuous nature of the pores are unknown in most practical cases. Under saturated conditions, $D_{p}$ can be approximated as:

$$
D_{p} \approx D_{a q} \theta
$$

Mechanical dispersion is a quantification of the scattering of particles in porous media due to fluctuations about the average velocity of the fluid. Classical parameterization of the mechanical dispersion coefficient is defined as a quantity linearly proportional to the velocity and it is assumed diffusion independent [22,23]. The commonly accepted form of the linear parametric model reads as [24]:

$$
D_{\text {mech }} \approx \alpha_{t} v
$$

where $\alpha_{t}$ is the transverse dispersivity, and $v$ is the absolute value of the seepage-velocity vector. $\alpha_{t}$ is a property of porous media, which in principle, can be measured [25]. A more accurate empirical parameterization of the mechanical dispersion based on an earlier statistical model [26] has been proposed and validated by two and three-dimensional well-controlled laboratory experiments [17,27-30], reads as:

$$
D_{\text {mech }}=D_{a q}\left(\frac{P e^{2}}{P e+2+4 \delta^{2}}\right)^{\beta}
$$

where $P e=v d / D_{a q}$ is the grain Péclet number, $d$ is the grain size, $\delta$ is the ratio between the length of a pore channel and its hydraulic radius, and $\beta$ is an empirical exponent that accounts for the degree of incomplete mixing within the pore channels [31,32]. A less-than-one $\beta$ value was found in the previous experiments $[17,27,29,30]$ and it is suggested to keep $\beta$ as a constant value $[17,27]$. The introduction of $\beta \neq 1$ is based on the hypothesis that the physical law for the growth rate of the volume occupies by a moving elementary mass of solute in a porous medium might be non-linear. Equation (7) implies a non-linear relationship between the mechanical dispersion coefficient and the flow velocity and a compound-dependency of the mechanical dispersion term [17,27]. Such a phenomenon has been observed in earlier experimental studies [33-39].

\section{Laboratory Experiments}

\subsection{Pore Scale}

Pore-scale experiments are rarely performed to study dilution and reactive mixing in solute transport. As indicated by Equation (3), only dynamic diffusion occurring at the pore spaces is included in the transport calculation. The dilution and reactive mixing processes can be explicitly 
quantified with low uncertainties by applying the detailed velocity data. Nevertheless, a few pore-scale experiments are still conducted to investigate the mixing and reaction kinetics in porous media [40-45]. At steady-state, the effects of grain sizes and structures and porous media heterogeneity on dilution and mixing-controlled reactions have been studied [46-48]. Here we briefly review the experiments performed at steady-state.

Pore-scale experiments have been commonly performed in two-dimensional flow-through micromodels. The standard lithographic technique is one among the others (e.g., Hele-Shaw, optical lithography, wet, dry and laser or plasma etching, stereo lithography [49]) frequently used to construct the geometry. The process is briefly described here. Silicon wafers are coated with a photoresist polymer and are selectively exposed to UV light by placing a mask above the wafer. The areas covered by the photoresist polymer are removed utilizing a developer, while the areas not covered by the photoresist polymer are etched using plasma generated from an inductively coupled plasma-deep reactive ion etching system. Flow channels are sealed by anodically bonding a cover sheet of Pyrex glass to etched silicon wafers. Details of the microfabrication process can be found in [47] and [50]. One representative example of the pore-scale experimental setup is shown in Figure 1. The length and width of a typical micromodel can be several centimeters. Solutions are usually injected through different channels with the help of pumps such that the mixing between the reactive solutions can be observed along the flow directions at the steady-state transport. Depending on different solutes used in the experiments, images of fluorescent intensity within the pore space are captured for the analysis of dilution and mixing results. A microscope equipped with an automated stage and a CCD camera (furnished in front of the micromodel) can facilitate the image captions controlled by NIS-Elements imaging software. The images are corrected for nonuniform illumination in post-processing.

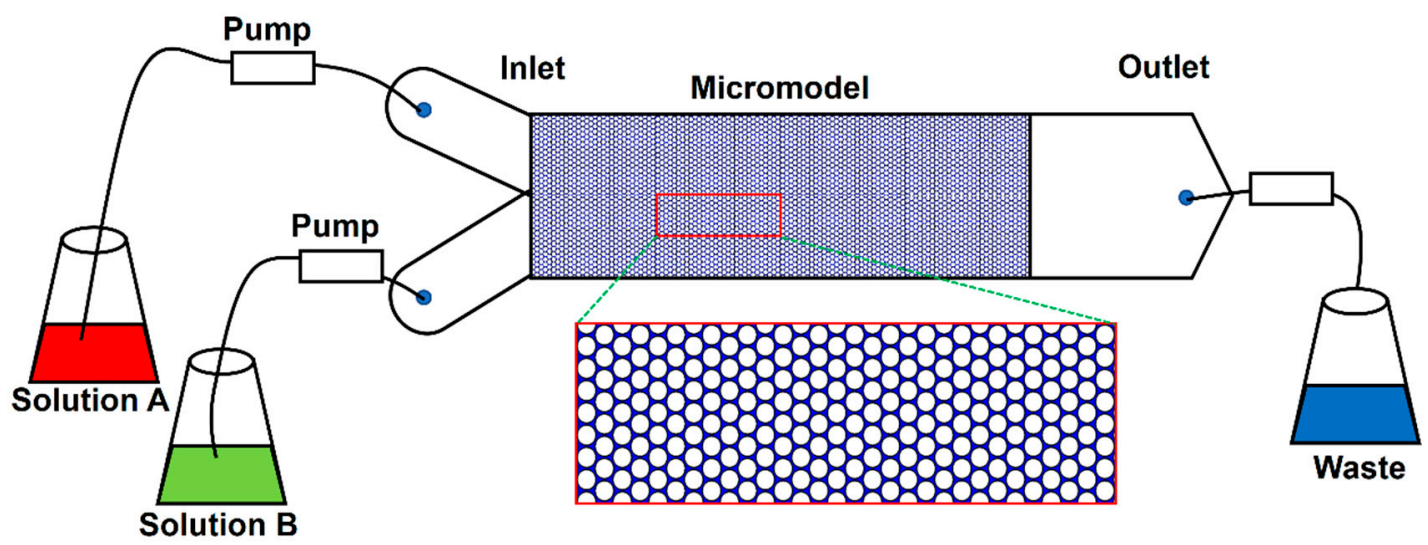

Figure 1. Pore-scale experimental setup with details of porous media structures: Solution A and Solution B are injected through two inlet ports; waste is extracted through outlet port; constant-rate injection and extraction are realized by three pumps.

Willingham et al. $[47,48]$ performed conservative and mixing-controlled reactive pore-scale experiments to investigate the effect of porous media structures on steady-state dilution and reactive mixing. Five different pore structures were considered, including cylinders and horizontal and vertical ellipses with different sizes and arrangements. Fluorophore Oregon Green Bapta 488 (OG5N) in the hexapotassium salt form and $\mathrm{Ca}^{2+}$ were used as solutes due to their fast reaction rate. They found that not only the grain size but also the grain orientation significantly affects mixing and reaction. Furthermore, flow focusing by bringing stream lines closer can increase the transverse concentration gradient thus enhance dilution and reactive mixing. The enhancement is greater in pore structures with longer flow focusing regions and a larger porosity contrast. Similar findings were also reported by Oostrom et al. [46] in which conservative solute Alexa 488 was used as the tracer in the pore-scale transport experiments. 
Pore-scale experiments are essential for the investigation of dilution and reactive mixing of solute transport. However, due to its difficulties of the experimental operation and low reproducibility, very few studies of two-dimensional and three-dimensional pore-scale experiments are reported to our knowledge. Instead, numerical simulations considering pore-scale dilution and reactive mixing have been constantly performed [51-54] and some of them are used for the interpretation of the experimental observations in Darcy-scale and field-scale transport problems $[28,46,47,55]$.

\subsection{Darcy Scale}

Darcy-scale experiments have been widely performed to study dilution and reactive mixing of solute transport in the last several decades [3,56-59]. In this section, we will first present the setups and techniques used in the experiments. Then we describe the steady-state dilution and reactive mixing considering conservative and reactive transport in homogeneous and heterogeneous porous media.

The setups performing flow-through experiments consist of one-, two- and three-dimensional systems. Since transverse dispersion is often the focus of the steady-state transport, two- and three-dimensional experiments are of general interest. In two-dimensional experiments only one transverse direction exists, while there are two transverse directions (i.e., horizontal and vertical) in three-dimensional flow-through systems. Three-dimensional experiments can better resemble the natural aquifer. However, due to their operational difficulties, three-dimensional experiments are seldom performed. Figure 2 shows a typical two-dimensional flow-through system. The two-dimensional flow-through chamber normally has a length and a width of around one meter. Note that the length and height of the system should be much larger than the width of the system to better resemble a two-dimensional system.

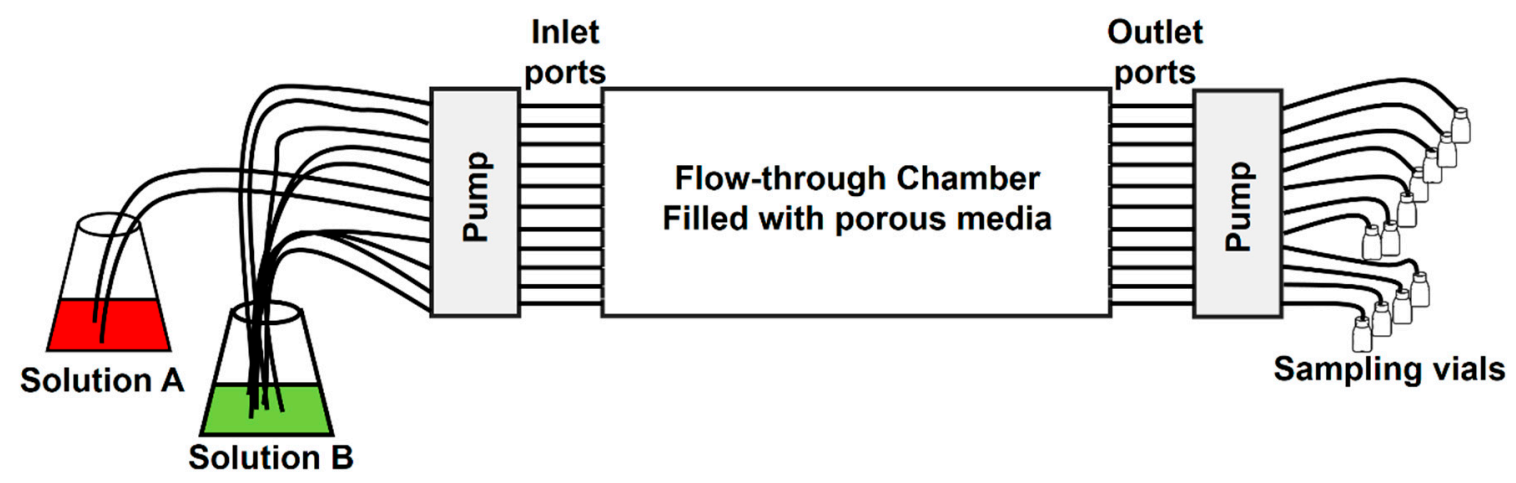

Figure 2. Two-dimensional Darcy-scale flow-through experimental setup: solutions are injected through inlet ports and extracted through outlet ports; constant-rate injection and extraction are realized by two high-precision pumps; samples are collected at the outlet channels.

Steady-state flow can be obtained by the constant head difference between the inlet and the outlet of the flow-through system or constant injection and extraction pumping rates at the inlet and outlet ports [30,60-63]. The former method needs an external injection of a second solution near the inlet and extraction of samples near the outlet. Such a procedure disturbs the flow field inside the porous media and is usually termed as invasive technique. The latter method (i.e., injection and extraction ports facilitate steady-state parallel flow as shown in Figure 2) is recommended here since the two solutions can be introduced simultaneously through the inlet without a disturbance of flow in the inner porous media. However, a high-resolution and high-precision measurements at the outlet requires many ports (i.e., many pumping channels) and high-precision pumps, thus increasing the labor and budgets of the experiments.

The materials filled in the flow-through systems are commonly glass beads or clean sands in order to exclude the influence of physical or (bio)chemical reactions at the surface of the porous medium. For the purpose of clear observation, glass beads are often used in the two-dimensional 
experiments. Index-matched solids and liquids phases were also used in the flow-through systems for the purpose of clearer quantification of flow fields and transport [64-66]. This technique is often limited to the specific solids and liquids. The liquids are often toxic and hazardous with few exceptions (e.g., hydrogel as solid matrix and water as liquid [67]). For quantitative analysis of plume distributions, non-invasive imaging methods were widely applied including optical imaging using UV or visible light [68,69], dual-energy gamma radiation [70,71], X-ray microtomography [72,73] and magnetic resonance imaging (MRI) [74,75]. Detailed theories and applications of the four imaging methods are referred to [76]. Optical methods have been most frequently used in the investigation of solute mixing since they are least expensive and easy to handle [77,78]. The only limitation is that they cannot facilitate three-dimensional imaging. Rather than the fluorescein intensity captured within the pore space in pore-scale experiments, the intensity captured in Darcy-scale experiments is macroscopic. In two-dimensional experiments, Haberer et al. [79] proposed a non-invasive optode technique that is based on the dynamic luminescence quenching of a luminophore by molecular oxygen to quantitatively measure the concentration of dissolved oxygen in the flow-through system. Ye et al. [80] qualitatively analyze solute distributions in three-dimensional inner porous media by freezing and slicing the materials after each experiment. Due to the slicing procedure, quantitative analysis of the plume distribution is not feasible. A direct and non-invasive method is to sample and measure the collected liquids at the outlet ports, however, what happened in the inner domain needs to be interpreted by numerical simulations $[17,27,81,82]$.

Mixing-controlled reactive transport was first proposed by numerical modeling assuming instantaneous reactions or double-Monod kinetic reactions between reaction partners $[7,10,14,83,84]$. Later, it was evidenced by laboratory experiments [15]. Bauer et al. [56,85,86] performed two-dimensional aerobic and anaerobic biodegradation experiments using toluene as the oxidizable compound and found that biodegradation activities were mainly located at the fringe of the toluene plumes.

The influence of the domain dimensionality on solute dilution was studied experimentally by Ye et al. [30]. Conservative tracers of fluorescein and oxygen were injected at the selected ports at the inlet and measured at the outlet ports. Even though transverse dispersion coefficients estimated from the two-dimensional and three-dimensional experiments are identical, dilution is stronger in three-dimensional systems compared to the two-dimensional systems. This is consistent with intuition since one more transverse direction is provided for dispersion and dilution.

Porous media heterogeneity has been verified experimentally to have a significant effect on dilution and mixing and reaction. In particular, it can facilitate a significant enhancement of dilution and reactive mixing of solute transport $[3,57,81,85,87]$. Groundwater streamlines converge and diverge in heterogeneous porous media, leading to the plume spreading and mixing. The spreading itself cannot enhance dilution or reactive mixing [88]. However, spreading can facilitate the enlargement of the material surface of the plume and thus increase transverse dispersion, dilution and reactive mixing. The enhancement of dilution and mixing by flow focusing and defocusing in high permeability inclusions was studied in both two-dimensional and three-dimensional systems $[3,81,85]$. An example of the three-dimensional heterogeneous porous media setup with high-permeability inclusions is shown in Figure 3a [81]. The distances between the neighboring streamlines are shortened due to the flow-focusing effect, beneficial transverse dispersion of the solutes in the inclusions. A second effect of flow focusing is that it accelerates the flow velocity and reduces the residence time of the solutes in the inclusions. In two-dimensional systems, the former positive effect for dilution and mixing overwhelms the latter one, leading to an enhancement of dilution and reactive mixing. However, the two effects counteract each other in three-dimensional systems. The theoretical analysis shows that there is no enhancement of dilution and reactive mixing by the flow-focusing effect if the transverse dispersion coefficient (i.e., $D_{t}$ ) is not velocity-dependent. However, $D_{t}$ depends on the velocity and grain size (refer to Equation (7)) and the correlation between hydraulic conductivity and grain size prevails over the enhancement of dilution and mixing in three-dimensional systems. Nevertheless, the enhancement of dilution and mixing by flow focusing is stronger in two-dimensional 
systems compared to three-dimensional systems. The detailed theoretical analysis can be referred to Werth et al. [89] and Ye et al. [81]. More importantly, the extent of dilution and mixing enhancement depends on the geometry of the porous medium, particularly the location of the high-permeability inclusions. Only if the plume fringe is focused in the high-permeability inclusions can dilution and mixing be significantly enhanced $[3,81]$.
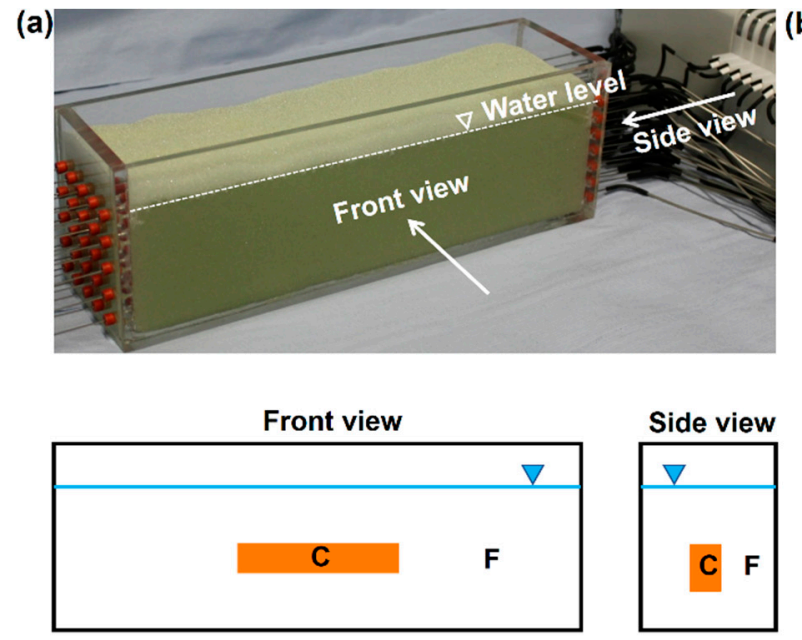
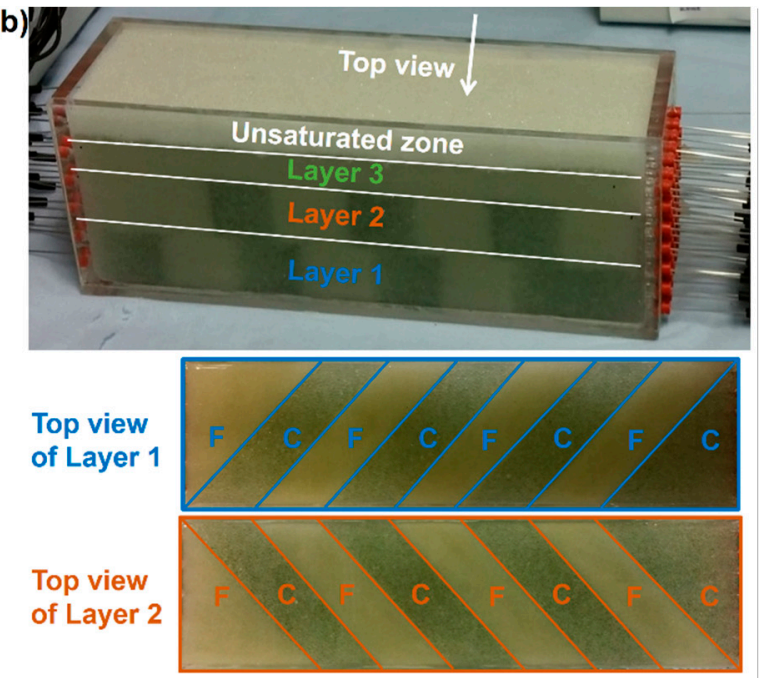

F: fine glass beads; C: coarse glass beads

Figure 3. (a) Heterogeneous isotropic porous media setup: three-dimensional overview, front view and side view. The orange cube represents the high-permeability inclusion with coarse glass beads, and the other porous media consist of fine glass beads (modified from [81]). (b) Macroscopic heterogeneous anisotropic porous media setup: three-dimensional overview and top view of Layer 1 and Layer 2. Layer 1 and Layer 2 are structured as simplified herringbone cross-stratification with alternated fine and coarse glass beads, Layer 3 and the unsaturated zone consist of fine glass beads (modified from $[80,82])$.

Macroscopic anisotropy of porous media has also been proved experimentally as an important effect influencing dilution and mixing of solute transport. An example of the experimental setup is shown in Figure $3 b[80,82]$. The streamlines twist creating helical flows in the porous media. Such a complex flow field leads to the deformation of the material surface of the plume and the enhancement of dilution and reactive mixing in porous media. Notice that the helical flow can only happen in three-dimensional flow-through systems. In two-dimensional systems, streamlines can only meander since two streamlines cannot intersect each other. However, due to the difficulties of experimental procedures and measuring techniques, very few experiments considering the effect of macroscopic anisotropic porous media on dilution were performed. Ye et al. [80,82] performed steady-state transport experiments using fluorescein as the conservative tracer solute and they measured the concentration at the outlet high-resolution extraction ports. However, plume distributions at the inner porous media were only measured qualitatively by freezing and slicing porous media after steady-state was reached. Nevertheless, sparse experimental data verified the numerical model and the numerical simulation helped interpret concentration distributions, dilution and reactive mixing both at the inner porous media and at the outlet of the flow-through system. More analytical and numerical studies were performed considering the effects of both microscopic and macroscopic anisotropic porous media on the helical flow, solute dilution and reactive mixing, and they showed the same trend as in the experimental outcomes [90-95].

Three-dimensional transverse dispersion and dilution were also studied in natural consolidated rock. Boon et al. [96] performed steady-state laboratory experiments using cylindrical Berea sandstone 
core as the material that plume transports. $\mathrm{NaI}$ was used as the conservative tracer and the plume distribution was observed by a medical X-ray CT scanner. They found that dilution was enhanced by the flow-focusing effect yet not significantly due to the homogeneity of the Berea sandstone.

\section{Quantification of Dilution and Reactive Mixing}

In the following section, parameters defined for the quantification of dilution and reactive mixing are introduced in conservative and reactive transport, respectively. Other parameters like the scalar dissipation rate $[17,19,97]$ used to describe the dilution and mixing extent are only used in modelling studies and not considered here.

\subsection{Conservative Transport}

\subsubsection{Moment Analysis}

The moment analysis has been widely used for the quantification of solute spreading in transport problems [98-101]. In steady-state transport with a continuously injected source, plume spreading at the transverse directions has been measured by the computation of the transverse spatial moment $[3,81,102]$.

In two-dimensional experiments, the solute concentration is considered constant at the very thin width (i.e., $W_{y}$ ) of the system and it varies along the height $\left(H_{z}\right)$. In this context, the normalized first transverse spatial moment (i.e., $m_{1}$ ) quantifies the location of the gravity center as a function of the longitudinal distance and can be calculated as:

$$
m_{1}(x)=\frac{\int_{0}^{W_{y}} \int_{0}^{H_{z}} z c(x, y, z) d y d z}{\int_{0}^{W_{y}} \int_{0}^{H_{z}} c(x, y, z) d y d z}
$$

The normalized second central transverse spatial moment (i.e., $m_{2 C}$ ) represents the spreading of the concentration distribution about its gravity center and its value can be calculated as:

$$
m_{2 C}(x)=\frac{\int_{0}^{W_{y}} \int_{0}^{H_{z}}\left(z-m_{1}(x)\right)^{2} c(x, y, z) d y d z}{\int_{0}^{W_{y}} \int_{0}^{H_{z}} c(x, y, z) d y d z}
$$

In three-dimensional experiments, the normalized first and second central transverse spatial moment at transverse longitudinal and vertical directions can be defined adopting an approach similar to Ye et al. [81]:

$$
\begin{gathered}
m_{1, y}(x)=\frac{\int_{0}^{W_{y}} y c_{y}(x, y) d y}{\int_{0}^{W_{y}} c_{y}(x, y) d y} \\
m_{1, z}(x)=\frac{\int_{0}^{H_{z}} z c_{z}(x, z) d z}{\int_{0}^{H_{z}} c_{z}(x, z) d z} \\
m_{2 C, y}(x)=\frac{\int_{0}^{W_{y}}\left(y-m_{1, y}(x)\right)^{2} c_{y}(x, y) d y}{\int_{0}^{W_{y}} c_{y}(x, y) d y} \\
m_{2 C, z}(x)=\frac{\int_{0}^{H_{z}}\left(z-m_{1, z}(x)\right)^{2} c_{z}(x, z) d z}{\int_{0}^{H_{z}} c_{z}(x, z) d z}
\end{gathered}
$$

where $m_{1, y}$ and $m_{1, z}$ are the normalized first transverse spatial moments at transverse longitudinal and vertical directions, $m_{2 C, y}$ and $m_{2 C, z}$ are the normalized second central transverse spatial moments 
at transverse longitudinal and vertical directions, and $c_{y}$ and $c_{z}$ represent the average concentrations along the transverse horizontal and vertical directions, respectively:

$$
\begin{aligned}
& c_{y}(x, y)=\frac{\int_{0}^{H_{z}} z c(x, y, z) d z}{H_{z}} \\
& c_{z}(x, z)=\frac{\int_{0}^{W_{y}} y c(x, y, z) d y}{W_{y}}
\end{aligned}
$$

The normalized second central transverse spatial moment quantifies dilution of the solute plume along the travel distance in homogeneous porous media setups. In homogeneous flow-through systems, both spreading and dilution are described by the local transverse dispersion coefficient. However, it could not correctly measure dilution in heterogeneous ones. In heterogeneous porous media solute plumes can squeeze and stretch, which is a purely advective movement of the plume and does not contribute to dilution directly [88,103]. Rolle et al. [3] has calculated $m_{2 C}$ in two-dimensional steady-state transport experiments. The value of $m_{2 \mathrm{C}}$ decreases once the plume enters into high-permeability inclusions. This phenomenon is not consistent with the natural rule that dilution decreases the peak concentration and increases the entropy of a conservative plume. A few other experiments also verify that the moment analysis can bias our understanding of dilution and mixing in heterogeneous porous media [104].

\subsubsection{Flux-Related Dilution Index}

The flux-related dilution index is a parameter proposed by Rolle et al. [3] to correctly quantify dilution of steady-state plumes with continuously emitted sources. It is based on the concept of the dilution index [88], which was derived for an analysis of dilution for a slug injection. Here, dilution was defined as "the act of distributing solute mass over a larger volume". In contrast, the flux-related dilution index was defined as "distributing a solute mass flux over a larger water flux" [3]. In the laboratory flow-through tank experiment, the flux-related dilution index (i.e., $E_{Q}$ ) can be written as:

$$
E_{Q}(x)=\exp \left[-\int_{0}^{H_{z}} \int_{0}^{W_{y}} p_{Q}(x, y, z) \ln \left(p_{Q}(x, y, z)\right) q_{x}(x, y, z) d y d z\right]
$$

where $q_{x}$ is the specific discharge in the longitudinal direction, and $p_{Q}$ is the flux-weighted probability density function of the solute mass defined as:

$$
p_{Q}(x, y, z)=\frac{c(x, y, z)}{\int_{0}^{H_{z}} \int_{0}^{W_{y}} c(x, y, z) q_{x}(x, y, z) d y d z}
$$

The upper limit of the flux-related dilution index is the total discharge passing through the system.

The flux-related dilution index has been applied in the experimental data analysis and shows a good measure of dilution in both homogeneous and heterogeneous porous media setups $[3,28,29,59,80-82]$. Figure 4 shows an example of the comparison of dilution measured at the inlet (i.e., $E_{Q, \text { inlet }}$ ) and outlet (i.e., $E_{Q, \text { outlet }}$ ) of the flow-through system. In both three-dimensional and two-dimensional systems, all the symbols are above the black line in Figure 4, indicating a larger dilution at the outlet compared to the inlet (i.e., $E_{Q, \text { outlet }}>E_{Q, \text { inlet }}$ ). Furthermore, dilution is enhanced by the heterogeneity and anisotropy of the porous media, which is reflected by the flux-related dilution index between homogeneous and heterogeneous anisotropic setups in Figure 4. 


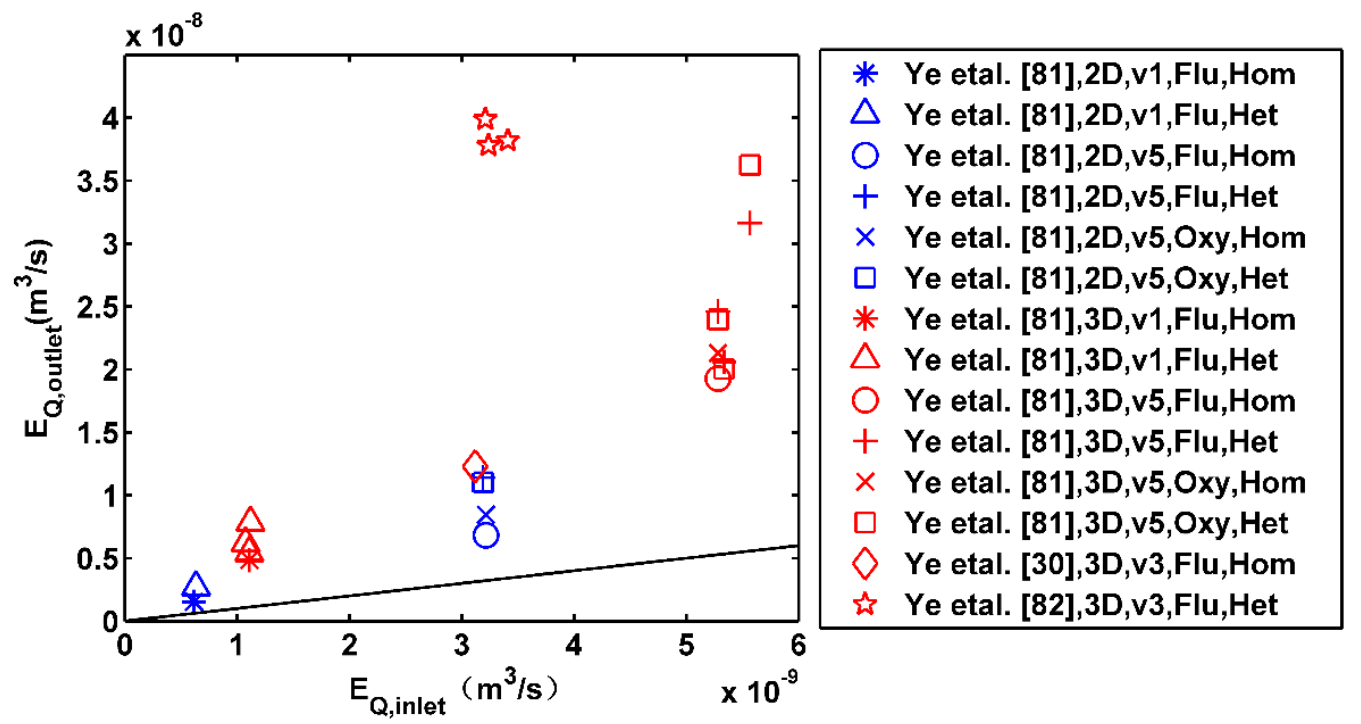

Figure 4. Flux-related dilution indices at the inlet and outlet for different experimental setups: the

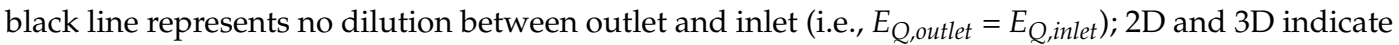
two-dimensional and three-dimensional flow-through systems respectively; v1, v3 and v5 represent flow velocities of $1 \mathrm{~m} /$ day, $3 \mathrm{~m} /$ day and $5 \mathrm{~m} /$ day respectively; Flu and Oxy represent a tracer solute of fluorescein and oxygen in conservative experiments respectively; Hom and Het represent homogenous and heterogeneous porous media.

A critical dilution index (i.e., $C D I$ ) is defined by Chiogna et al. [1,17] to estimate the dilution necessary for a complete degradation of a steady-state reactive plume. Its value equals the flux-related dilution index at the cross-section where the reactive plume ends up. In the application of the experiments, once a reactive plume length is observed, dilution can be quantified by the calculation of the flux-related dilution index of a conservative plume at that cross-section. Indeed, the CDI is a parameter relating dilution between a conservative and a reactive compound.

\subsection{Reactive Transport}

\subsubsection{Plume Length}

In mixing-controlled steady-state reactive transport experiments, plume length is the most intuitive parameter quantifying the extent of transverse mixing $[3,28,78]$. Here the plume length (i.e., $L$ ) is defined as the distance between the continuously released source and the downstream end of the reactive plume. In flow-through experiments, the concentration of the reactive solutions has to be selected properly such that the plume ends up within the tank. A shorter plume indicates a relatively better transverse mixing and reaction. For instance, flow focusing in high permeability inclusions leads to an enhanced transverse mixing, resulting to a significantly shorter plume length compared to that in the homogeneous porous media setup [3].

The plume length can also be directly related to a more popular parameter, $D_{t}$. In cases that one reactive solution $(A)$ is injected as a line source or square source and the other reactive compound $(B)$ was injected in the ambient solution, the effective transverse dispersion coefficient (i.e., $D_{t, e f f}$ ) can be estimated from the observed plume length of $A$ in two- and three-dimensional systems respectively, defined as $[80,105]$ :

$$
\begin{gathered}
\text { 2D : } D_{t, e f f}=\frac{v h^{2}}{16 L\left[\text { inver } f\left(X_{\text {crit }}\right)\right]^{2}} \\
\text { 3D : } D_{t, e f f}=\frac{v h^{2}}{16 L\left[\text { inverf }\left(\sqrt{X_{\text {crit }}}\right)\right]^{2}}
\end{gathered}
$$


where $h$ is the height of the injected reactive source, and $X_{\text {crit }}$ is the critical mixing ratio according to [105]. Two such equations were derived from the analytical solutions of the advection-dispersion equation in homogeneous setups. Therefore, $D_{t, \text { eff }}$ might be considerably larger than the local transverse dispersion coefficient when the plume length is measured in a heterogeneous porous media setup.

\subsubsection{Mass Flux}

The product or reactant mass flux is also an intuitive measure of transverse mixing in mixing-controlled steady-state reactive transport experiments [3,28,85]. At the outlet of the flow-through system, flow velocities at the extraction ports can be easily obtained by collecting samples at the defined time interval. Thus, the product mass flux can be computed with the measured concentration and the flow velocity at the outlet. However, measuring the mass flux in the inner porous media is not feasible since the flow rate cannot be easily measured in the inner flow-through system without interference of the flow field. Nevertheless, it is still a useful parameter applied in some studies. Rolle et al. [3] has found that the product mass flux is much larger at the outlet of the heterogeneous porous media setups compared to the homogeneous one, indicating a mixing enhancement by the flow-focusing effect in heterogeneous high-permeability inclusions. Bauer et al. [85] has reported a smaller mass flux of the reactant plume in the heterogeneous setup than in the homogeneous setup due to mixing and the biodegradation enhancement by porous media heterogeneity.

\section{Summary and Outlook}

This paper briefly reviews the experimental works studying steady-state dilution and reactive mixing in saturated porous media. Starting from the theory behind the experiments, we have reviewed laboratory experimental setups and results obtained at the pore and the Darcy scales. In this context, we have described the experimental techniques used for the observation of the plume distribution and the measurement of concentrations and flow velocities. Some limitations and difficulties of the experimental techniques have been mentioned. For instance, steady-state parallel flow can be obtained only with very high-precision pumps, which are relatively expensive. The impact of heterogeneity of the porous medium has been emphasized. Finally, the parameters commonly applied for the quantification of dilution and reactive mixing have been described briefly. The classical moment analysis measures spreading instead of real dilution of the solute plumes, which has to be considered particularly for the correct quantification of dilution in heterogeneous porous media. The flux-related dilution index is a useful property quantifying steady-state dilution and mixing in both homogeneous and heterogeneous flow-through experiments.

In order to better understand dilution and reactive mixing processes in natural aquifers, more laboratory experiments with complex setups are necessary. Suggested future works are summarized as follows:

1. In most performed experiments, glass beads and clean sands were used as the porous medium to exclude the external effects such as the irregular shapes of the grains and the sorption of the compounds on the grain surfaces. However, natural porous media indeed has irregular shapes and physical and chemical sorption potentials. Such characteristics of the natural porous media have additional effects on solute dilution and mixing. In future laboratory experiments, we suggest more applications of the porous medium taken from the natural field. To simplify the experimental conditions, we could pre-treat the porous medium. For instance, we could sieve and classify the grains if the target of our research interest is the influence of the grain sizes and shapes on dilution and reactive mixing.

2. The experimental studies have shown the importance of single or several high-permeability inclusions and macroscopic anisotropic lenses to dilution and reactive mixing. As to the larger scale, Di Dato et al. [106] performed numerical simulations to calculate the influence of multiple heterogeneous microstructures on macrodispersion and showed a high relevance between macrodispersion and heterogeneous microstructures. However, no experiments have 
ever been performed to test the different spatial distributions of multiple inclusions and lenses on large-scale dilution and mixing. In future laboratory experiments, we suggest properly enlarging the scale of the flow-through domain and including more heterogeneous lenses to resemble spatial features observed in the natural aquifers.

3. So far, most experiments were performed involving conservative transport or very simple reactions. However biogeochemical reactive transport is the common phenomenon in natural aquifer systems. Even though complex reactive transport is rather difficult to operate in laboratory experiments, it should be tested to help better understand reactive mixing.

4. Hydrodynamic dispersion, particularly mechanical dispersion, has an impact on the structure of fingers in density-driven nature convection. Recent experimental studies found that hydrodynamic dispersion enhances the merging of fingers and thus reduces the finger numbers [107-109]. Such a process indicates an effect of hydrodynamic dispersion on dilution and reactive mixing of solute fingers in density-driven natural convection. Therefore, density effects may also be considered and included in the future investigation of dilution and reactive mixing laboratory experiments.

5. By complicating the experimental conditions, more advanced experimental operation and measurement techniques are required. Particularly in the three-dimensional systems, development of an easy and cheap method for observation and quantification of plume distributions in the inner porous media would help dramatically advance our understanding of dilution and reactive mixing from an experimental perspective. Therefore, during future experimental studies, development of better experimental methods and techniques could also be a key point to break through experimental studies of dilution and reactive mixing.

In conclusion, our final target is not only to understand a simple mechanism inducing and influencing dilution and reactive mixing but also to resemble and understand what happens in the natural aquifer systems. Therefore, we might combine different physical and biogeochemical processes in experiments to check if the experimental results are a superposition of every single mechanism and to further verify the numerical models we have used for field transport predictions.

Author Contributions: Study Design, T.X., Y.Y. and Y.X.; Literature Search, T.X., Y.Y., Y.Z. and Y.X.; Writing-Original Draft Preparation, T.X., Y.Y. and Y.Z.; Figure Preparation, T.X. and Y.Y.; Investigation, T.X. and Y.Z.; Revision, T.X., Y.Y. and Y.X.; Supervison, Y.Y.; Funding Acquisition, Y.Y. and Y.X.

Funding: This research was funded by National Natural Science Foundation of China $(51709085,51679067$ and 41702243) and the Fundamental Research Funds for the Central Universities (2017B00214).

Conflicts of Interest: The authors declare no conflict of interest.

\section{References}

1. Chiogna, G.; Cirpka, O.A.; Grathwohl, P.; Rolle, M. Transverse mixing of conservative and reactive tracers in porous media: Quantification through the concepts of flux-related and critical dilution indices. Water Resour. Res. 2011, 47, W02505. [CrossRef]

2. Chiogna, G.; Hochstetler, D.L.; Bellin, A.; Kitanidis, P.K.; Rolle, M. Mixing, entropy and reactive solute transport. Geophy. Res. Lett. 2012, 39, L20405. [CrossRef]

3. Rolle, M.; Eberhardt, C.; Chiogna, G.; Cirpka, O.A.; Grathwohl, P. Enhancement of dilution and transverse reactive mixing in porous media: Experiments and model-based interpretation. J. Contam. Hydrol. 2009, 110, 130-142. [CrossRef]

4. Mace, R.E.; Fisher, R.S.; Welch, D.M.; Parra, S.P. Extent, Mass, and Duration of Hydrocarbon Plumes from Leaking Petroleum Storage Tank Sites in Texas; Geologic Circular 97-1; Bureau of Economic Geology, University of Texas at Austin: Austin, TX, USA, 1997.

5. Wiedemeier, T.H.; Rifai, H.S.; Newell, C.J.; Wilson, J.T. Natural Attenuation and Fuels and Chlorinated Solvents in the Subsurface; John Wiley \& Sons, Inc.: Hoboken, NJ, USA, 1999.

6. Anneser, B.; Einsiedl, F.; Meckenstock, R.U.; Richters, L.; Wisotzky, F.; Griebler, C. High-resolution monitoring of biogeochemical gradients in a tar oil-contaminated aquifer. Appl. Geochem. 2008, 23, 1715-1730. [CrossRef] 
7. Cirpka, O.A.; Frind, E.O.; Helmig, R. Numerical simulation of biodegradation controlled by transverse mixing. J. Contam. Hydrol. 1999, 40, 159-182. [CrossRef]

8. Ham, P.A.S.; Schotting, R.J.; Prommer, H.; Davis, G.B. Effects of hydrodynamic dispersion on plume lengths for instantaneous bimolecular reactions. Adv. Water Resour. 2004, 27, 803-813. [CrossRef]

9. Knutson, C.; Valocchi, A.; Werth, C. Comparison of continuum and pore-scale models of nutrient biodegradation under transverse mixing conditions. Adv. Water Resour. 2007, 30, 1421-1431. [CrossRef]

10. Mayer, K.U.; Benner, S.G.; Frind, E.O.; Thornton, S.F.; Lerner, D.N. Reactive transport modeling of processes controlling the distribution and natural attenuation of phenolic compounds in a deep sandstone aquifer. J. Contam. Hydrol. 2001, 53, 341-368. [CrossRef]

11. Prommer, H.; Anneser, B.; Rolle, M.; Einsiedl, F.; Griebler, C. Biogeochemical and isotopic gradients in a BTEX/PAH contaminant plume: Model-based interpretation of a high-resolution field data set. Environ. Sci. Technol. 2009, 43, 8206-8212. [CrossRef]

12. Amos, R.T.; Berkins, B.A.; Delin, G.N.; Cozzarelli, I.M.; Blowes, D.W.; Kirshtein, J.D. Methane oxidation in a crude oil contaminated aquifer: Delineation of aerobic reactions at the plume fringes. J. Contam. Hydrol. 2011, 125, 13-25. [CrossRef]

13. Davis, G.B.; Barber, C.; Power, T.R.; Thierrin, J.; Patterson, B.M.; Rayner, J.L.; Wu, Q. The variability and intrinsic remediation of a BETX plume in anaerobic sulphate-rich groundwater. J. Contam. Hydrol. 1999, 36, 265-290. [CrossRef]

14. Thornton, S.F.; Quigley, S.; Spence, M.J.; Banwart, S.A.; Bottrell, S.; Lerner, D.N. Processes controlling the distribution and natural attenuation of dissolved phenolic compounds in a deep sandstone aquifer. J. Contam. Hydrol. 2001, 53, 233-267. [CrossRef]

15. Tuxen, N.; Albrechtsen, H.-J.; Bjerg, P.L. Identification of a reactive degradation zone at a landfill leachate plume fringe using high resolution sampling and incubation techniques. J. Contam. Hydrol. 2006, 85, 179-194. [CrossRef]

16. Dentz, M.; Le Borgne, T.; Englert, A.; Bijeljic, B. Mixing, spreading and reaction in heterogeneous media: A brief review. J. Contam. Hydrol. 2011, 120, 1-17. [CrossRef]

17. Chiogna, G. Transverse Mixing of Conservative and Reactive Tracers in Porous Media. Ph.D. Thesis, University of Tübingen, Tübingen, Germany, 2010.

18. Cirpka, O.A.; de Barros, F.P.J.; Chiogna, G.; Rolle, M.; Nowak, W. Stochastic flux-related analysis of transverse mixing in two-dimensional heterogeneous porous media. Water Resour. Res. 2011, 47, WR010279. [CrossRef]

19. Chiogna, G.; Cirpka, O.A.; Grathwohl, P.; Rolle, M. Relevance of local compound-specific transverse dispersion for conservative and reactive mixing in heterogeneous porous media. Water Resour. Res. 2011, 47, W07540. [CrossRef]

20. Boving, T.B.; Grathwohl, P. Tracer diffusion coefficients in sedimentary rocks correlation to porosity and hydraulic conductivity. J. Contam. Hydrol. 2001, 53, 85-100. [CrossRef]

21. Grathwohl, P. Diffusion in Natural Porous Media: Contaminant Transport, Sorption/Desorption, and Dissolution Kinetics; Kluwer Academic Publishers: Boston, MA, USA, 1998.

22. De Josselin de Jong, G. Longitudinal and transverse diffusion in granular deposits. EOS Trans. AGU 1958, 39, 67-74. [CrossRef]

23. Saffman, P.G. A theory of dispersion in a porous medium. J. Fluid Mech. 1959, 6, 321-349. [CrossRef]

24. Scheidegger, A.E. General theory of dispersion in porous media. J. Geophys. Res. 1961, 66, 3273-3278. [CrossRef]

25. Scheidegger, A.E. Statistical hydrodynamics in porous media. J. Appl. Phys. 1954, 25, 994-1001. [CrossRef]

26. Bear, J.; Bachmat, Y. A Generalized Theory on Hydrodynamic Dispersion in Porous Media. In Proceedings of the IASH Symposium on Artificial Recharge and Management of Aquifers, Haifa, Israel, 1967; Available online: http:/ / hydrologie.org/redbooks/a072/07201.pdf (accessed on 20 October 2018).

27. Chiogna, G.; Eberhardt, C.; Grathwohl, P.; Cirpka, O.A.; Rolle, M. Evidence of compound-dependent hydrodynamic and mechanical transverse dispersion by multitracer laboratory experiments. Environ. Sci. Technol. 2010, 44, 688-693. [CrossRef]

28. Hochstetler, D.L.; Rolle, M.; Chiogna, G.; Haberer, C.M.; Grathwohl, P.; Kitanidis, P.K. Effects of compound-specific transverse mixing on steady-state reactive plumes: Insights from pore-scale simulations and Darcy-scale experiments. Adv. Water Resour. 2013, 54, 1-10. [CrossRef] 
29. Rolle, M.; Hochstetler, D.; Chiogna, G.; Kitanidis, P.K.; Grathwohl, P. Experimental investigation and pore-scale modeling interpretation of compound-specific transverse dispersion in porous media. Transp. Porous Media 2012, 93, 347-362. [CrossRef]

30. Ye, Y.; Chiogna, G.; Cirpka, O.A.; Grathwohl, P.; Rolle, M. Experimental investigation of compound-specific dilution of solute plumes in saturated porous media: 2-D vs. 3-D flow-through systems. J. Contam. Hydrol. 2015, 172, 33-47. [CrossRef]

31. Chiogna, G.; Bellin, A. Analytical solution for reactive solute transport considering incomplete mixing within a reference elementary volume. Water Resour. Res. 2013, 49, 2589-2600. [CrossRef]

32. Tartakovsky, A.M.; Tartakovsky, G.D.; Scheibe, T.D. Effects of incomplete mixing on multicomponent reactive transport. Adv. Water Resour. 2009, 32, 1674-1679. [CrossRef]

33. Blackwell, R.J. Laboratory studies of microscopic dispersion phenomena. Soc. Petrol. Engrs. J. 1962, 225, 1-8. [CrossRef]

34. Carvalho, J.F.; Delgado, J.M.P.Q. Lateral dispersion in liquid flow through packed beds at $\mathrm{Pe}<1400$. Am. Inst. Chem. Eng. 2000, 46, 1089-1095. [CrossRef]

35. Delgado, J.M.P.Q.; Carvalho, J.F.R. Measurement of the coefficient of transverse dispersion in flow through packed beds for a wide range of values of the Schmidt number. Transp. Porous Media 2001, 44, 165-180. [CrossRef]

36. Harleman, D.R.F.; Rumer, R.R. Longitudinal and lateral dispersion in an isotropic porous medium. J. Fluid Mech. 1964, 16, 385-394. [CrossRef]

37. Huang, W.E.; Oswald, S.E.; Lerner, D.N.; Smith, C.C.; Zheng, C.M. Dissolved oxygen imaging in a porous medium to investigate biodegradation in a plume with limited electron acceptor supply. Environ. Sci. Technol. 2003, 37, 1905-1911. [CrossRef] [PubMed]

38. Klenk, I.D.; Grathwohl, P. Transverse vertical dispersion in groundwater and the capillary fringe. J. Contam. Hydrol. 2002, 58, 111-128. [CrossRef]

39. Olsson, Å.; Grathwohl, P. Transverse dispersion of non-reactive tracers in porous media: A new nonlinear relationship to predict dispersion coefficients. J. Contam. Hydrol. 2007, 92, 149-161. [CrossRef] [PubMed]

40. de Anna, P.; Jimenez-Martinez, J.; Tabuteau, H.; Turuban, R.; Le Borgne, T.; Derrien, M.; Meheust, Y. Mixing and reaction kinetics in porous media: An experimental pore scale quantification. Environ. Sci. Technol. 2014, 48, 508-516. [CrossRef] [PubMed]

41. Fanizza, M.F.; Yoon, H.; Zhang, C.; Oostrom, M.; Wietsma, T.W.; Hess, N.J.; Bowden, M.E.; Strathmann, T.J.; Finneran, K.T.; Werth, C.J. Pore-scale evaluation of uranyl phosphate precipitation in a model groundwater system. Water Resour. Res. 2013, 49, 874-890. [CrossRef]

42. Ghaderi, S.M.; Kharrat, R.; Tahmasebi, H.A. Experimental and theoretical study of calcium sulphate precipitation in porous media using glass micromodel. Oil Gas Sci. Technol. 2009, 64, 489-501. [CrossRef]

43. Nambi, I.M.; Werth, C.J.; Sanford, R.A.; Valocchi, A.J. Pore-scale analysis of anaerobic halorespiring bacterial growth along the transverse mixing zone of an etched silicon pore network. Environ. Sci. Technol. 2003, 37, 5617-5624. [CrossRef] [PubMed]

44. Yoon, H.; Valocchi, A.J.; Werth, C.J.; Dewers, T. Pore-scale simulation of mixing-induced calcium carbonate precipitation and dissolution in a microfluidic pore network. Water Resour. Res. 2012, 48, W02524. [CrossRef]

45. Zhang, C.Y.; Dehoff, K.; Hess, N.; Oostrom, M.; Wietsma, T.W.; Valocchi, A.J.; Fouke, B.W.; Werth, C.J. Pore-scale study of transverse mixing induced $\mathrm{CaCO} 3$ precipitation and permeability reduction in a model subsurface sedimentary system. Environ. Sci. Technol. 2010, 44, 7833-7838. [CrossRef]

46. Oostrom, M.; Mehmani, Y.; Romero-Gomez, P.; Tang, Y.; Liu, H.; Yoon, H.; Kang, Q.; Joekar-Niasar, V.; Balhoff, M.T.; Dewers, T.; et al. Pore-scale and continuum simulations of solute transport micromodel benchmark experiments. Computat. Geosci. 2016, 20, 857-879. [CrossRef]

47. Willingham, T.W.; Werth, C.J.; Valocchi, A.J. Evaluation of the effects of porous media structure on mixing-controlled reactions using pore-scale modeling and micromodel experiments. Environ. Sci. Technol. 2008, 42, 3185-3193. [CrossRef] [PubMed]

48. Willingham, T.W.; Zhang, C.; Werth, C.J.; Valocchi, A.J.; Oostrom, M.; Wietsma, T.W. Using dispersivity values to quantify the effects of pore-scale flow focusing on enhanced reaction along a transverse mixing zone. Adv. Water Resour. 2010, 33, 525-535. [CrossRef]

49. Karadimitriou, N.K.; Hassanizadeh, S.M. A review of micromodels and their use in two-phase flow studies. Vadose Zone J. 2012, 11. [CrossRef] 
50. Chomsurin, C.; Werth, C.J. Analysis of pore-scale nonaqueous phase liquid dissolution in etched silicon pore networks. Water Resour. Res. 2003, 39, WR001643. [CrossRef]

51. Acharya, R.C.; Valocchi, A.J.; Werth, C.J.; Willingham, T.W. Pore-scale simulation of dispersion and reaction along a transverse mixing zone in two-dimensional porous media. Water Resour. Res. 2007, 43, W10435. [CrossRef]

52. Alhashmi, Z.; Blunt, M.J.; Bijeljic, B. Predictions of dynamic changes in reaction rates as a consequence of incomplete mixing using pore scale reactive transport modeling on images of porous media. J. Contam. Hydrol. 2015, 179, 171-181. [CrossRef]

53. Rolle, M.; Chiogna, G.; Hochstetler, D.L.; Kitanidis, P.K. On the importance of diffusion and compound-specific mixing for groundwater transport: An investigation from pore to field scale. J. Contam. Hydrol. 2013, 153, 51-68. [CrossRef]

54. Rolle, M.; Kitanidis, P.K. Effects of compound-specific dilution on transient transport and solute breakthrough: A pore-scale analysis. Adv. Water Resour. 2014, 71, 186-199. [CrossRef]

55. Porta, G.M.; Chaynikov, S.; Thovert, J.F.; Riva, M.; Guadagnini, A.; Adler, P.M. Numerical investigation of pore and continuum scale formulations of bimolecular reactive transport in porous media. Adv. Water Resour. 2013, 62, 243-253. [CrossRef]

56. Bauer, R.D.; Maloszewski, P.; Zhang, Y.C.; Meckenstock, R.U.; Griebler, C. Mixing-controlled biodegradation in a toluene plume-Results from two-dimensional laboratory experiments. J. Contam. Hydrol. 2008, 96, 150-168. [CrossRef] [PubMed]

57. Castro-Alcalá, E.; Fernandez-Garcia, D.; Carrera, J.; Bolster, D. Visualization of mixing processes in a heterogeneous sand box aquifer. Environ. Sci. Technol. 2012, 46, 3228-3235. [CrossRef] [PubMed]

58. McNeil, J.D.; Oldenborger, G.A.; Schincariol, R.A. Quantitative imaging of contaminant distributions in heterogeneous porous media laboratory experiments. J. Contam. Hydrol. 2006, 84, 36-54. [CrossRef] [PubMed]

59. Muniruzzaman, M.; Rolle, M. Experimental investigation of the impact of compound-specific dispersion and electrostatic interactions on transient transport and solute breakthrough. Water Resour. Res. 2017, 53, 1189-1209. [CrossRef]

60. Haberer, C.M.; Rolle, M.; Cirpka, O.A.; Grathwohl, P. Impact of heterogeneity on oxygen transfer in a fluctuating capillary fringe. Groundwater 2013, 53, 57-70. [CrossRef] [PubMed]

61. Katz, G.E.; Berkowitz, B.; Guadagnini, A.; Saaltink, M.W. Experimental and modeling investigation of multicomponent reactive transport in porous media. J. Contam. Hydrol. 2011, 120, 27-44. [CrossRef] [PubMed]

62. Levy, M.; Berkowitz, B. Measurement and analysis of non-Fickian dispersion in heterogeneous porous media. J. Contam. Hydrol. 2003, 64, 203-226. [CrossRef]

63. Rolle, M.; Muniruzzaman, M.; Haberer, C.M.; Grathwohl, P. Coulombic effects in advection-dominated transport of electrolytes in porous media: Multicomponent ionic dispersion. Geochim. Cosmochim. Acta 2013, 120, 195-205. [CrossRef]

64. Amini, N.; Hassan, Y.A. An investigation of matched index of refraction technique and its application in optical measurements of fluid flow. Exp. Fluids 2012, 53, 2011-2020. [CrossRef]

65. Rashidi, M.; Peurrung, L.; Tompson, A.F.B.; Kulp, T.J. Experimental analysis of pore-scale flow and transport in porous media. Adv. Water Resour. 1996, 19, 163-180. [CrossRef]

66. Stöhr, M.; Roth, K.; Jähne, B. Measurement of 3D pore-scale flow in index-matched porous media. Exp. Fluids 2003, 35, 159-166. [CrossRef]

67. Rubol, S.; Tonina, D.; Vincent, L.; Sohm, J.A.; Basham, W.; Budwig, R.; Savalia, P.; Kanso, E.; Capone, D.G.; Nealson, K.H. Seeing through porous media: An experimental study for unveiling interstitial flows. Hydrol. Proc. 2018, 32, 402-407. [CrossRef]

68. Huang, W.E.; Smith, C.C.; Lerner, D.N.; Thornton, S.F.; Oram, A. Physical modelling of solute transport in porous media, evaluation of an imaging technique using UV excited fluorescent dye. Water Res. 2002, 36, 1843-1853. [CrossRef]

69. Zinn, B.; Meigs, L.C.; Harvey, C.F.; Haggerty, R.; Peplinski, W.J.; Schwerin, C.F. Experimental visualization of solute transport and mass transfer processes in two-dimensional conductivity fields with connected regions of high conductivity. Environ. Sci. Technol. 2004, 38, 3916-3926. [CrossRef] [PubMed] 
70. Hofstee, C.; Oostrom, M.; Dane, J.H.; Walker, R.W. Infiltration and redistribution of perchloroethylene in partially saturated, stratified porous media. J. Contam. Hydrol. 1998, 34, 293-313. [CrossRef]

71. Oostrom, M.; Dane, J.H.; Guven, O.; Hayworth, J.S. Experimental investigation of dense solute plumes in an unconfined aquifer model. Water Resour. Res. 1992, 28, 2315-2326. [CrossRef]

72. Betson, M.; Barker, J.; Barnes, P.; Atkinson, T. Use of synchrotron tomographic techniques in the assessment of diffusion parameters for solute transport in groundwater flow. Transp. Porous Media 2005, 60, 217-223. [CrossRef]

73. Perret, J.; Prasher, S.O.; Kantzas, A.; Langford, C. A two-domain approach using CAT scanning model solute transport in soil. J. Environ. Qual. 2000, 29, 995-1010. [CrossRef]

74. Amin, M.H.G.; Chorely, R.J.; Richards, K.S.; Hall, L.D.; Carpenter, T.A.; Cislerova, M.; Vogel, T. Study of infiltration into a heterogeneous soil using magnetic resonance imaging. Hydrol. Proc. 1997, 11, 471-483. [CrossRef]

75. Deurer, M.; Vogeler, I.; Clothier, B.E.; Scotter, D.R. Magnetic resonance imaging of hydrodynamic dispersion in a saturated porous medium. Transp. Porous Media 2004, 54, 145-166. [CrossRef]

76. Werth, C.J.; Zhang, C.; Brusseau, M.L.; Oostrom, M.; Baumann, T. A review of non-invasive imaging methods and applications in contaminant hydrogeology research. J. Contam. Hydrol. 2010, 113, 1-24. [CrossRef] [PubMed]

77. Oates, P.M.; Harvey, C.F. A colorimetric reaction to quantify fluid mixing. Exp. Fluids 2006, 41, 673-683. [CrossRef]

78. Rahman, M.A.; Jose, S.C.; Nowak, W.; Cirpka, O.A. Experiments on vertical transverse mixing in a large-scale heterogeneous model aquifer. J. Contam. Hydrol. 2005, 80, 130-148. [CrossRef] [PubMed]

79. Haberer, C.M.; Rolle, M.; Liu, S.; Cirpka, O.A.; Grathwohl, P. A high-resolution non-invasive approach to quantify oxygen transport across the capillary fringe and within the underlying groundwater. J. Contam. Hydrol. 2011, 122, 26-39. [CrossRef] [PubMed]

80. Ye, Y.; Chiogna, G.; Cirpka, O.A.; Grathwohl, P.; Rolle, M. Experimental investigation of transverse mixing in porous media under helical flow conditions. Phys. Rev. E 2016, 94, 013113. [CrossRef] [PubMed]

81. Ye, Y.; Chiogna, G.; Cirpka, O.A.; Grathwohl, P.; Rolle, M. Enhancement of plume dilution in two-dimensional and three-dimensional porous media by flow focusing in high-permeability inclusions. Water Resour. Res. 2015, 51, 5582-5602. [CrossRef]

82. Ye, Y.; Chiogna, G.; Cirpka, O.A.; Grathwohl, P.; Rolle, M. Experimental evidence of helical flow in porous media. Phys. Rev. Lett. 2015, 115, 194502. [CrossRef] [PubMed]

83. Prommer, H.; Barry, D.A.; Davis, G.B. Modelling of physical and reactive processes during biodegradation of a hydrocarbon plume under transient groundwater flow conditions. J. Contam. Hydrol. 2002, 59, 113-131. [CrossRef]

84. Prommer, H.; Tuxen, N.; Bjerg, P. Fringe-controlled natural attenuation of phenoxy acids in a landfill plume: Integration of field-scale processes by reactive transport modeling. Environ. Sci. Technol. 2006, 40, 4732-4738. [CrossRef]

85. Bauer, R.D.; Rolle, M.; Bauer, S.; Eberhardt, C.; Grathwohl, P.; Kolditz, O.; Meckenstock, R.U.; Griebler, C. Enhanced biodegradation by hydraulic heterogeneities in petroleum hydrocarbon plumes. J. Contam. Hydrol. 2009, 105, 56-68. [CrossRef]

86. Bauer, R.D.; Rolle, M.; Kürzinger, P.; Grathwohl, P.; Meckenstock, R.U.; Griebler, C. Two-dimensional flow-through microcosms-Versatile test systems to study biodegradation processes in porous aquifers. J. Hydrol. 2009, 369, 284-295. [CrossRef]

87. Fox, D.T.; Guo, L.J.; Fujita, Y.; Huang, H.; Redden, G. Experimental and numerical analysis of parallel reactant flow and transverse mixing with mineral precipitation in homogeneous and heterogeneous porous media. Transp. Porous Media 2016, 111, 605-626. [CrossRef]

88. Kitanidis, P.K. The concept of the dilution index. Water Resour. Res. 1994, 30, 2011-2026. [CrossRef]

89. Werth, C.J.; Cirpka, O.A.; Grathwohl, P. Enhanced mixing and reaction through flow focusing in heterogeneous porous media. Water Resour. Res. 2006, 42, W12414. [CrossRef]

90. Chiogna, G.; Rolle, M.; Alberto, B.; Cirpka, O.A. Helicity and flow topology in three-dimensional anisotropic porous media. Adv. Water Resour. 2014, 73, 134-143. [CrossRef]

91. Chiogna, G.; Cirpka, O.A.; Rolle, M.; Alberto, B. Helical flow in three-dimensional nonstationary anisotropic heterogeneous porous media. Water Resour. Res. 2015, 51, 261-280. [CrossRef] 
92. Cirpka, O.A.; Chiogna, G.; Rolle, M.; Bellin, A. Transverse mixing in three-dimensional nonstationary anisotropic heterogeneous porous media. Water Resour. Res. 2015, 51, 241-260. [CrossRef]

93. Hemker, K.; Baker, M. Analytical solutions for whirling groundwater flow in two-dimensional heterogeneous anisotropic aquifers. Water Resour. Res. 2006, 42, W12419. [CrossRef]

94. Stauffer, F. Impact of highly permeable sediment units with inclined bedding on solute transport in aquifers. Adv. Water Resour. 2007, 30, 2194-2201. [CrossRef]

95. Ye, Y.; Chiogna, G.; Lu, C.; Rolle, M. Effect of anisotropy structures on plume entropy and reactive mixing in helical flows. Transp. Porous Media 2018, 121, 315-332. [CrossRef]

96. Boon, M.; Bijeljic, B.; Niu, B.; Krevor, S. Observations of 3-D transverse dispersion and dilution in natural consolidated rock by X-ray tomography. Adv. Water Resour. 2016, 96, 266-281. [CrossRef]

97. Bolster, D.; Benson, D.A.; Le Borgne, T.; Dentz, M. Anomalous mixing and reaction induced by superdiffusive nonlocal transport. Phys. Rev. E 2010, 82, 021119. [CrossRef] [PubMed]

98. Adams, E.E.; Gelhar, L.W. Field study of dispersion in a heterogeneous aquifer: 2. Spatial moments analysis. Water Resour. Res. 1992, 28, 3293-3307. [CrossRef]

99. Dagan, G. Transport in heterogeneous porous formations: Spatial moments, ergodicity, and effective dispersion. Water Resour. Res. 1990, 26, 1281-1290. [CrossRef]

100. Harvey, C.F.; Gorelick, S.M. Temporal moment-generating equations: Modeling transport and mass transfer in heterogeneous aquifers. Water Resour. Res. 1995, 31, 1895-1911. [CrossRef]

101. Valocchi, A.J. Spatial moment analysis of the transport of kinetically adsorbing solutes through stratified aquifers. Water Resour. Res. 1989, 25, 273-279. [CrossRef]

102. Ballarini, E.; Sauer, S.; Eberhardt, C.; Beyer, C. Evaluation of the role of heterogeneities on transverse mixing in bench-scale tank experiments by numerical modeling. Groundwater 2014, 52, 368-377. [CrossRef]

103. Dentz, M.; Carrera, J. Mixing and spreading in stratified flow. Phys. Fluids 2007, 19, 017107. [CrossRef]

104. Ursino, N.; Gimmi, T.; Flühler, H. Dilution of non-reactive tracers in variably saturated sandy structures. Adv. Water Resour. 2001, 24, 877-885. [CrossRef]

105. Cirpka, O.A.; Olsson, Å.; Ju, Q.; Rahman, M.A.; Grathwohl, P. Determination of transverse dispersion coefficients from reactive plume lengths. Groundwater 2006, 44, 212-221. [CrossRef]

106. Di Dato, M.; de Barros, F.P.J.; Fiori, A.; Bellin, A. Effects of the hydraulic conductivity microstructure on macrodispersivity. Water Resour. Res. 2016, 52, 6818-6832. [CrossRef]

107. Wang, L.; Nakanishi, Y.; Hyodo, A.; Suekane, T. Three-dimensional structure of natural convection in a porous media: Effect of dispersion on finger structure. Int. J. Greenh. Gas Control 2016, 53, 274-283. [CrossRef]

108. Nakanishi, Y.; Hyodo, A.; Wang, L.; Suekane, T. Experimental study of 3D Rayleigh-Taylor convection between miscible fluids in a porous medium. Adv. Water Resour. 2016, 97, 224-232. [CrossRef]

109. Liang, Y.; Wen, B.; Hesse, M.A.; DiCarlo, D. Effect of dispersion on solutal convection in porous media. Geophy. Res. Lett. 2018, 45, 9690-9698. [CrossRef] 